\title{
Dynamic Evolution of Securities Market Network Structure under Acute Fluctuation Circumstances
}

\author{
Haifei Liu, ${ }^{1}$ Tingqiang Chen, ${ }^{2}$ and Zuhan $\mathrm{Hu}^{1}$ \\ ${ }^{1}$ School of Management and Engineering, Nanjing University, Nanjing 210093, China \\ ${ }^{2}$ School of Economic and Management, Nanjing Tech University, Nanjing 211816, China \\ Correspondence should be addressed to Tingqiang Chen; tingqiang88888888@163.com
}

Received 2 June 2017; Revised 27 August 2017; Accepted 14 September 2017; Published 6 November 2017

Academic Editor: Dimitri Volchenkov

Copyright (C) 2017 Haifei Liu et al. This is an open access article distributed under the Creative Commons Attribution License, which permits unrestricted use, distribution, and reproduction in any medium, provided the original work is properly cited.

This empirical research applies cointegration in the traditional measurement method first to build directed weighted networks in the context of stock market. Then, this method is used to design the indicators and the value simulation for measuring network fluctuation and studying the dynamic evolution mechanism of stock market transaction networks as affected by price fluctuations. Finally, the topological structure and robustness of the network are evaluated. The results show that network structure stability is strong in the bull market stage and weak in the bear market stage. And the convergence rate of the dynamic evolution of network fluctuation is higher in the bull market stage than in the bear market stage.

\section{Introduction}

In the financial market, individual and institutional investors, listed companies, and trading systems have self-organizing characteristics. Mutual relationships between investors, investment banks and financial analysts, investment funds and listed companies, and so forth form the network topological structure of financial securities market. Network structure plays an important role in information transmission and risk contagion. The higher the network concentration of securities, the higher the volatility of securities. Moreover, network structure influences the learning mechanisms of investors. That is, investors often use different aspects of their social networks to update investment beliefs and make decisions. In addition, network structure has a certain influence on the decision-making and corporate governance of listed companies. Network structure can be used to reflect capital market volatility and stability. Therefore, depicting the network structure of securities fluctuation is a feasible way of analyzing the influence of random factors on the macro behavior of the financial market.

Network structure has been applied in the financial market via the complex network theory and method. But network structure cannot give play to the advantages of traditional research methods, and it is difficult to analyze and measure the fluctuation of economics using the simple network analysis methods. Therefore, the current study employs cointegration in the traditional measurement method to build a directed weighted network of the stock market and design the indicator and the value simulation for measuring the fluctuation of this network. We aim to study the dynamic evolution mechanism of the stock market transaction network in a state of fluctuation and thereby evaluate the topological structure and robustness of the network. The methods used in this paper have been researched and empirically tested in the Shanghai-Hong Kong Stock Connect market and defined by different time windows.

The remainder of this paper is structured as follows. Section 2 provides a review of and commentary on previous, relevant literature. Section 3 describes the research method and model building and expounds on the specific methods used for the analysis of stock market stability. Section 4 presents the empirical analysis based on sample stocks from the Shanghai-Hong Kong Stock Connect market. Section 5 provides the research conclusions.

\section{Related Literatures}

With the continued development of the financial sector, the ties among market entities have become closer in terms 
of breadth and depth and have formed a complex system. Benefitting from the introduction and deepening of the complex network theory, the complex financial system method can best be described through comparison with traditional measurement methods. The internal structure of financial systems posits that while the latter may not always be stable, they can be relatively stable over a longer period. Financial and social networks have similar degrees of distribution and small world effects. Using the social network analysis method can thus help develop a new recognition of the relationship between nodes in the financial network (government, institutions, and individuals) and provide guidance to the regulation of financial reform and financial risk $[1,2]$. To date, studies on complex finance networks have mainly involved their description, as well as the analysis of their stability and degree to which they could carry the contagion of financial risk.

In describing financial networks, researchers have built network structure and explored the nature of the network statistics of different financial institutions [3-7], stock markets [8-10], international stock markets [11], crude oil future and spot markets [12], open-end fund markets [13], and mutual fund markets [14]. For example, Huang et al. [15] constructed a financial network of China's SH 180 Index and SZ 100 Index constituent stocks and explored the correlations among the stocks. Most descriptions and analyses of stock transaction networks indicate that the tail-end of the degree distribution of stock transaction networks has the characteristics of the power-law distribution [16]. Wang [17] constructed a weighted stock network by taking the secondboard market in China's Shanghai securities market as the data source and researching the stock network's "rich group" coefficient. He found the clear existence of such a "rich group" by exploring the random network with the same distribution degree.

Some works used the complex network theory to investigate the contagion of global financial system (Kali et al., 2009; Gai and Kapadia, 2010; Chen et al., 2016) [2, 18, 19], established a network model for the global transactions system, and studied global communications with regard to financial crises by measuring the indicators of network correlation and centrality among others $[4,19,20]$. To study the interactive influence mechanism among interfinance risk, some works established a financial network model that enabled them to analyze the robustness of a financial system on the basis of the complex network theory (Gai and Kapadia, 2010) [19], and analyzed the common characteristics of the optimal financial network and the topological structure of actual financial networks [19]. Some scholars further studied the behavior of a network structure in financial contagion and proposed relevant measures to improve the stability of a financial system from the financial network angle by preparing policy guidance for preventing crisis-level contagion $[2,4,7,19]$ (Wu et al., 2014; Chen et al., 2016). For example, Winecoff [21] studied the influence of global financial crises on politics and economics from a macro angle by establishing a complex network model. In addition, some works studied the stability of financial network. For example, Tumminello et al. [22] employed the MST and PMFG algorithms to build a complex network of 300 stocks from the New York Stock Exchange and found that the lower the data frequency, the more prominent the network hierarchy is. Huang et al. [23] used a threshold method to construct the financial network structure of China's stock market by analyzing the nature and topological stability and studying the topological robustness of the network and found that while the financial network had certain robustness against a random attack, it was brittle against a calculated attack. Lin et al. [24] employed MST and PMFG to build and analyze a financial market network by dynamically highlighting the key node in the network, studied the network stability, and certified that it is robust in terms of recognizing the key node using the centrality of the complex network node. Vitali et al. [25] dynamically analyzed the relationship between network nodes by establishing a financial network model between banks and companies, analyzing the indicators of the network's topological structure, and studying the network's degree of invulnerability. Our aims are to integrate the econometrics model with the building of a complex financial market network and design relevant indicators to weigh the robustness of this network dynamically.

\section{Research Method and Model Building}

3.1. Building a Directed Weighted Network. The linear combination of two or more nonstationary time series may be of stationary or low-order integration. In an actual financial market, certain time series that are not stationary exist and may cause inconvenience in the analysis, but others are stationary (equal-average, finite variance, and autocorrelation coefficient is the function of lag intervals for endogenous) and follow the linear combination. From a financial perspective, when different nonstationary time series turn into stationary ones following a linear combination, equilibrium exists between these series. The relationship, which is known as cointegration, is stable on a long-term basis. In financial measurement, cointegration can describe the stable longterm relationship between two or more series. Therefore, this paper proposes building a directed weighted network based on the cointegration between stock time series.

Generally, to test the cointegration of two time series, we use the Engle-Granger two-step method based on Yang et al. [26] and Tu [27]. Suppose $x_{t}$ and $y_{t}$ are the logarithm return series of two stocks, where

$$
\begin{aligned}
& x_{t}=\ln \left(p_{x}(t)\right)-\ln \left(p_{x}(t-1)\right), \\
& y_{t}=\ln \left(p_{y}(t)\right)-\ln \left(p_{y}(t-1)\right),
\end{aligned}
$$

where $x_{t}$ denotes the restoration of the right price of stock $x$ at time $t$ and $y_{t}$ denotes the restoration of the right price of stock $y$ at time $t$.

Then, the cointegration test of $x_{t}$ and $y_{t}$ should be performed by supposing the equation

$$
y_{t}=\alpha+\tau x_{t}+u_{t},
$$

where $\alpha$ represents intercept term of the above long-term cointegration equation, $\tau$ is defined as regression coefficient, and $u_{t}$ is disequilibrium error.

If the number of stocks is $N$, two-two stock pairs can be formed completely by $N(N-1) / 2$. In this case, the 
cointegration test should be performed $N(N-1) / 2$ times to determine whether a connection exists between the two stocks. If a stock pair fails to pass the cointegration test, then the relationship that exists between the two stocks is not stable and a corresponding connection exists.

For every group of stock pairs that pass the cointegration test, we build an error correction model and use the firstorder lag series to judge the causality and determine the direction of the connection. The regression coefficient obtained from the equation serves as the weight of the side.

$$
\begin{aligned}
& \Delta y_{t}=\beta_{0}+\beta_{1} \cdot \Delta x_{t-1}+\beta_{2} \cdot u_{t-1}+\varepsilon_{t}, \\
& \Delta y_{t}^{\prime}=\beta_{0}^{\prime}+\beta_{1}^{\prime} \cdot \Delta x_{t-1}+\beta_{2}^{\prime} \cdot u_{t-1}+\varepsilon_{t}^{\prime} .
\end{aligned}
$$

If $\beta_{1}$ in (3) is significant but $\beta_{1}^{\prime}$ in (4) is insignificant, the logarithm return of stock $x$ will influence the logarithm return of stock $y$. Meanwhile, the logarithm return of stock $y$ will have no influence on the logarithm return of stock $x$. Hence, the directions of Nodes $x$ and $y$ in the network structure are such that Node $x$ points to Node $y$, and the weight is $\beta_{1}$. If $\beta_{1}$ in (3) and $\beta_{1}^{\prime}$ in (4) are significant, then a relationship of mutual influence exists between the logarithm return of stock $x$ and the logarithm return of stock $y$. In the network, Nodes $x$ and $y$ are bidirectional, and their weights are $\beta_{1}$ and $\beta_{1}^{\prime}$, respectively.

The weight of edge of the network $\beta$ constitutes the network's connection matrix $(D)$. If $w_{i j}=0$, Node $i$ does not point to Node $j$. The in- and out-strength of every node are then calculated using

$$
\begin{gathered}
S_{\text {in }}=\sum_{j \in V_{\text {in }}} w_{j i}, \\
S_{\text {iout }}=\sum_{j \in V_{\text {iout }}} w_{i j},
\end{gathered}
$$

where $w_{i j}$ refers to the weight that Node $i$ points to Node $j$, $V_{\text {iout }}$ means that $i$ is close to and points to the Node $i$ set, and $V_{i \text { in }}$ means that Node $i$ is close to and points to the Node $i$ set.

Given $K$ nodes with maximum strength and allowing for a certain degree of fluctuation in each node, the fluctuation of every $\beta$ spreads along the side of the network. Studying the fluctuation of the whole network over time enables the evaluation of the stability of the whole network.

3.2. Analysis Matrix of Network Stability. Supposing that the stock returns of all nodes in the network are $R_{0}=$ $\left[R_{01}, R_{02}, \ldots, R_{0 n}\right]$ at time 0 , and creating a certain disturbance in the network node at a certain time that yields $\Delta R_{0}=$ $\left[\Delta R_{01}, \Delta R_{02}, \ldots, \Delta R_{0 n}\right]$, the range of variation of $\Delta R$ will be $[-10 \%, 10 \%]$ due to the price limits in China's stock market. To explore the influence of $\Delta R_{0}$ on network node yield $R_{1}$ at Moment 1, we define the first-order effect matrix $W$ as follows:

$$
W=\left[\begin{array}{cccc}
0 & w_{12} & \cdots & w_{1 n} \\
w_{21} & 0 & \cdots & w_{2 n} \\
\cdots & \cdots & \cdots & \cdots \\
w_{n 1} & w_{n 2} & \cdots & 0
\end{array}\right]
$$

The first-order effect matrix $W$ refers to the first-order lag effect weight if the yield of $R_{i, t-1}$ has no influence on the yield of $R_{i, t}$ when $i=j, w_{i j}=0$. Under other circumstances, when $i$ has influence over $j, w_{i j}=\beta_{1}$. If $i$ is irrelevant to $j, w_{i j}=0$.

Supposing there is an influence of every stock node on the other stock nodes in the network, then the relationship can be superimposed in a linear manner, as expressed by the following equation:

$$
\begin{aligned}
\Delta R_{t, i} & \\
= & \frac{\left(\Delta R_{t-1,1} \times w_{1 i}+\Delta R_{t-1,2} \times w_{2 i}+\cdots+\Delta R_{t-1, n} \times w_{n i}\right)}{\sum_{j=1}^{n}\left|w_{j i}\right|} \\
= & \frac{1}{\sum_{j=1}^{n}\left|w_{j i}\right|} \\
& \cdot\left[\begin{array}{llll}
\Delta R_{t-1,1} & \Delta R_{t-1,2} & \cdots & \Delta R_{t-1, n}
\end{array}\right]\left[\begin{array}{llll}
w_{1 i} & w_{2 i} & \cdots & w_{n i}
\end{array}\right]^{T} .
\end{aligned}
$$

If changed into the matrix format, then the relationship takes the following form:

$$
\begin{aligned}
R_{t}= & R_{t-1} \times W \\
& \times\left[\begin{array}{cccc}
\frac{1}{\sum_{j=1}^{n} w_{j 1}} & 0 & \cdots & 0 \\
0 & \frac{1}{\sum_{j=1}^{n} w_{j 2}} & \ldots & 0 \\
\ldots & \cdots & \cdots & \ldots \\
0 & 0 & 0 & \frac{1}{\sum_{j=1}^{n} w_{j n}}
\end{array}\right] .
\end{aligned}
$$

To study the dynamic evolution characteristics of the network yield disturbance $\Delta R_{0}=\left[\Delta R_{01}, \Delta R_{02}, \ldots, \Delta R_{0 n}\right]$ at time 0 over time, this study designed the measurement indicator based on the weight-related matrix.

$$
\begin{aligned}
C_{t} & =\left(1+\Delta \bar{R}_{0}\right) \times\left(1+\Delta \bar{R}_{1}\right) \times \cdots \times\left(1+\Delta \bar{R}_{n}\right) \\
& =\prod_{i=0}^{n}\left(1+\Delta \bar{R}_{i}\right),
\end{aligned}
$$

where $\Delta \bar{R}_{i}$ refers to the arithmetic mean of $\Delta R_{i}$. Indicator $C_{t}$ rejects spontaneous change over the time of the yield of the stock node. The result could only explore the influence of the network's yield disturbance $\Delta R_{0}=\left[\Delta R_{01}, \Delta R_{02}, \ldots, \Delta R_{0 n}\right]$ at time 0 on the yield of the entire network through networkbased communication.

\section{Empirical Research and Analysis}

4.1. Data Source and Variable Selection. The China Securities Regulatory Commission and the Securities and Futures Commission of Hong Kong jointly issued a proclamation on April 10, 2014. On November 17, 2014, the transaction was officially opened. This study obtained data of 884 stocks from the WIND database as the initial sample, which consisted of 568 Shanghai-to-Hong Kong and 316 Hong Kong-toShanghai trading stocks. The sample series duration taken 


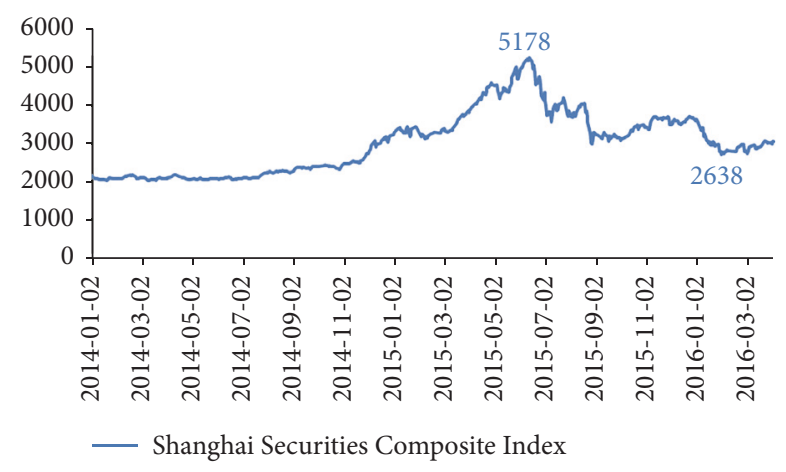

FIGURE 1: Trend of Shanghai Composite Index.

was November 17, 2014, to January 27, 2016. During this period, the Shanghai Composite Index reached 5,178 on June 12, 2015, but later fell steeply and decreased to 2,638 on January 27, 2016. We then divided the time window of the sample series into two sections. The first section was from November 17, 2014, to June 12, 2015, and was referred to as the "pre-stock market disaster period." The second section was from 12th June 2015 to 2nd January, 2016, and was referred to as the "post-stock market disaster period." After rejecting the 44 stocks listed later and in suspension, the remaining 840 stocks were used as the final sample (Figure 1).

4.2. Topological Structure of the Network. Table 1 shows that, in terms of all indicators, the values prior to the stock market disaster are lower than those following the disaster, indicating that the postcrisis network structure became more integrated, whereas the precrisis network structure was more dispersed.

The investigation of the in/out-strength distribution of the topological network structure of the Shanghai-Hong Kong Stocks before and after stock market disaster showed that regardless of the in- and out-strength distribution, the overall strength distribution was more extensive following the crisis. For example, prior to the stock market disaster, the instrengths of most nodes were under 50, and the maximum was higher than 100. Conversely, after the disaster, a large portion of the in-strengths exceeded 100 and even reached 200 (as shown in Figure 2(a)). Prior to the crisis, the outstrengths of most nodes were under 60 , and the maximum was 70. After the disaster, a large portion of the in-strengths exceeded 150 and reached as high as 250 (as shown in Figure 2(b)). The latter results indicated that the network structure of the Shanghai-Hong Kong Stock Connect market became more integrated after the stock market disaster. Consequently, neither the in- nor the out-strength fell within either the Poisson or normal distribution, with a very fat tail and clear power-law distribution characteristics. The latter indicated that, in the Shanghai-Hong Kong Stock Connect market, nodes exist with very high in- and out-strengths, which are extensively influenced by other nodes.

Likewise, in view of the in/out-degree distribution of the topological network structure of Shanghai-Hong Kong Stock Connect sample objects before and after the stock market disaster, the distribution degree was found more extensive following the crisis regardless of either the in- or the out-degree distribution. For example, before the crisis, the in-degree of most nodes was under 300, and the maximum was 400 . In contrast, a high proportion of the in-degrees of the nodes exceeded 400 after the crisis, reaching as high as 600 (as shown in Figure 2(d)). Before the stock market disaster, most out-degrees were under 200, and the maximum was 300 . After the stock market disaster, a large portion of the out-degrees exceeded 400, reaching 600 (as shown in Figure 2(e)). These results indicate that after the stock market disaster, the network structure of the Shanghai-Hong Kong Stock Connect market became more closely interlinked. In addition, the power-law characteristics of the degree distribution were similar to the characteristics of strength distribution.

4.3. Market Network Structure Stability. To calculate the network stability, we arranged the network nodes according to their out-strength by first selecting the degree $K$ of nodes and giving them disturbance $\Delta R_{0}=\left[\Delta R_{01}, \Delta R_{02}, \ldots, \Delta R_{0 n}\right]$. For ease of analysis, we supposed that $\Delta R_{01}=\Delta R_{02}=\cdots=$ $\Delta R_{0 n}=\phi$, where $\phi$ took four different values: $-10 \%,-5 \%, 5 \%$, and $10 \%$, and the degree $K$ is equal to 10 .

Figure 3 shows that fluctuation may tend to converge over time, which also indicates that the value of weighing fluctuation $C_{t}$ of the entire network converges to a fixed value. In line with this, the amplitude of fluctuation after the stock market disaster can be expected to exceed that prior to the disaster. We found that when the initial fluctuation was positive, the amplitude spread over the network was mostly positive. Conversely, when the initial fluctuation was negative, the amplitude spread over the network was mostly negative. This indicates that the direction of the initial fluctuation determines the direction of the dynamic evolution of fluctuation across the entire network.

Figure 4 shows that the direction of initial fluctuation determines the direction of the follow-up dynamic evolution of the network. A negative initial fluctuation makes $C_{t}$ in the network change to a negative direction, and vice versa. However, after a time, the fluctuation may converge. Before the stock market disaster, the fluctuation tended to converge after 6 to 7 iterations, whereas after the stock market disaster, the fluctuation tended to converge after 22 to 25 iterations. At the same time, the network's $C_{t}$ change in amplitude was very low before the stock market disaster, but increased after the stock market disaster. The latter shows that the network structure before the stock market disaster was dispersed and quick to digest external disturbances. By contrast, after the stock market disaster, the network structure was more integrated, but with more systematic risks, and the network structure was unable to digest external disturbances quickly.

4.4. Network Structure Divided by the Time Window and Its Stability. We divided the entire time window of the study into three stages, with the first-order stage and the previous analysis unchanged (i.e., from November 17, 2014, to June 12, 2015). This stage represented the rising bull market. The steep-fall period was then redivided into two stages, with stage 1 of steep-fall time from June 12, 2015, to August 25, 

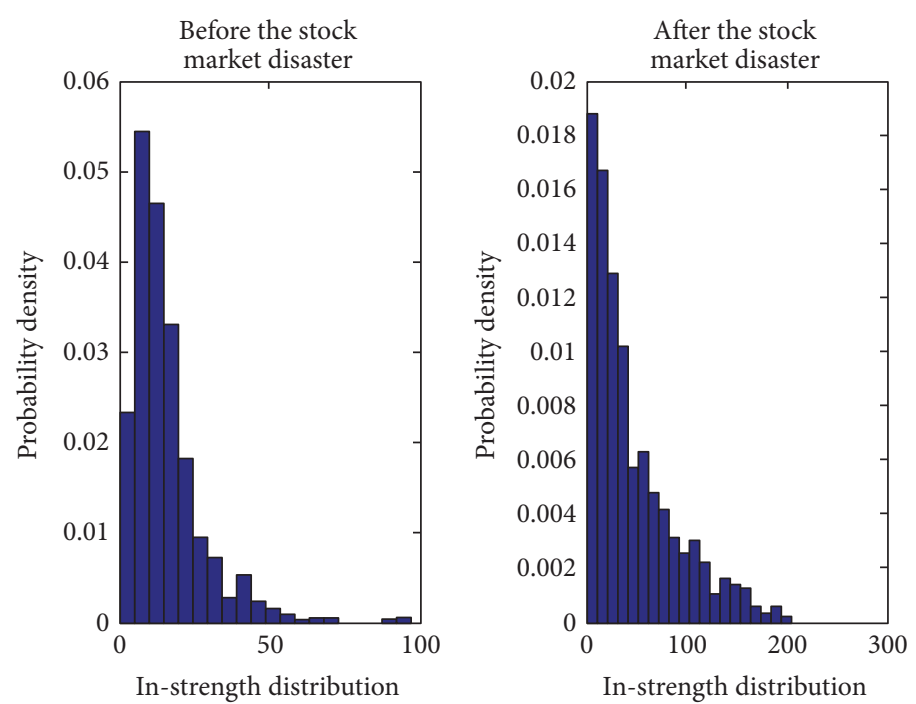

(a) In-strength distribution of topological network before and after the stock market disaster
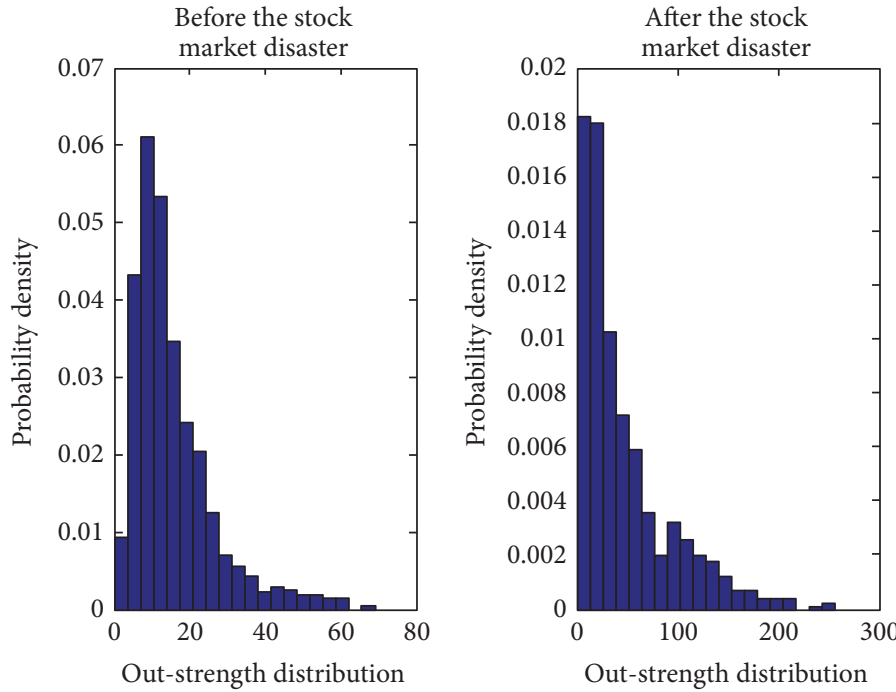

(b) Out-strength distribution of topological network before and after the stock market disaster
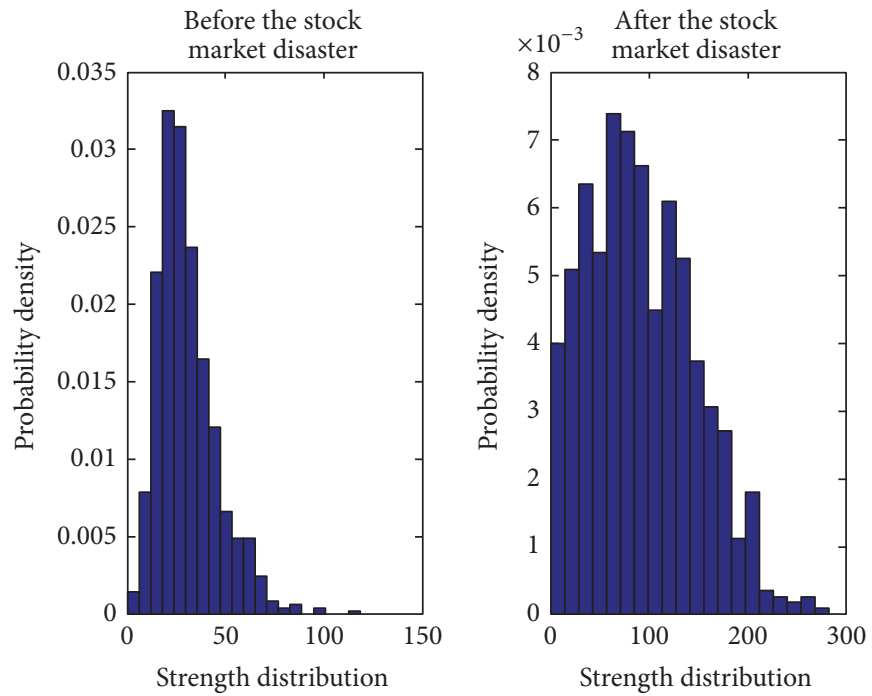

(c) Strength distribution of topological network structure before and after the stock market disaster

FIgURe 2: Continued. 

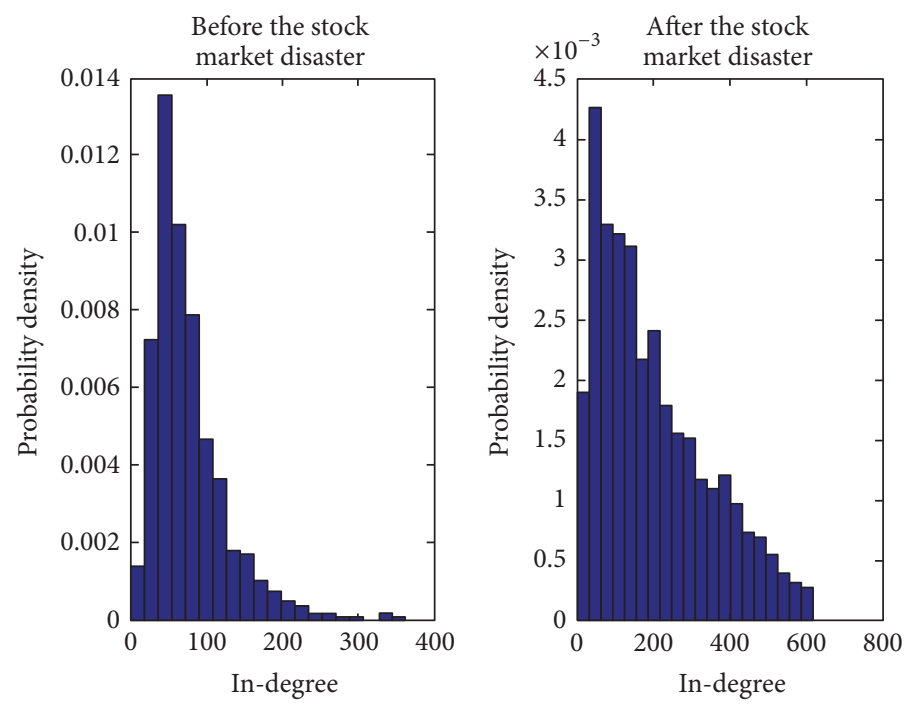

(d) In-degree of topological network before and after the stock market disaster
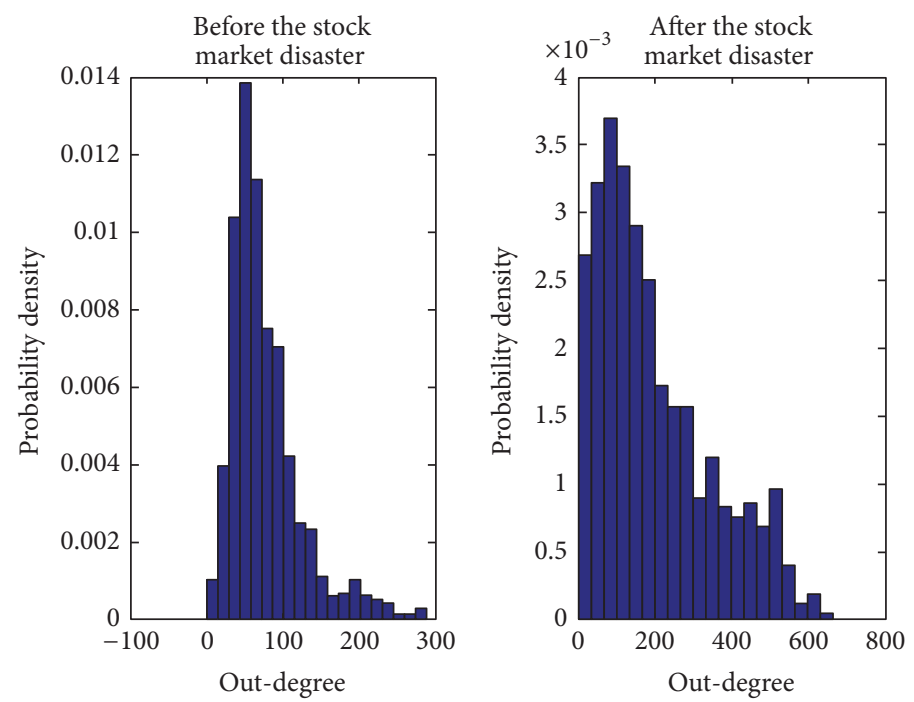

(e) Out-degree of topological network before and after the stock market disaster
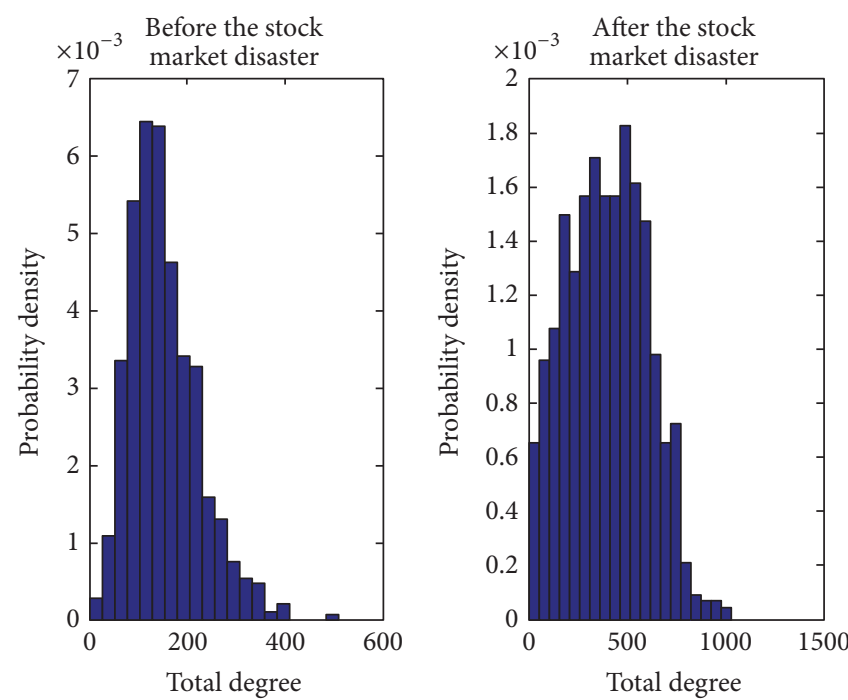

(f) Total degree of topological network structure before and after the stock market disaster

FIGURE 2: Strength distribution and degree distribution before and after the stock market disaster. 

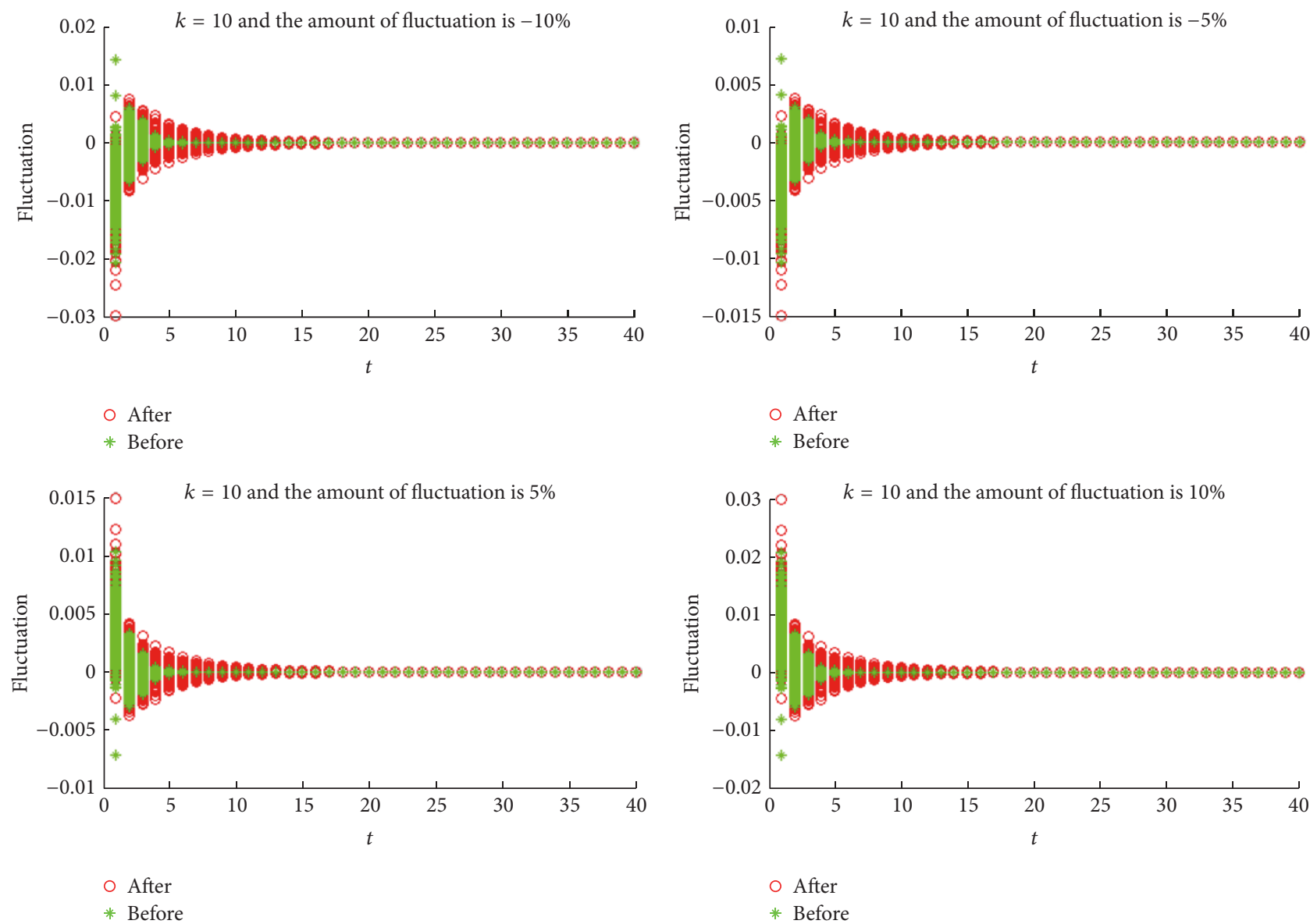

Figure 3: Dynamic evolution of fluctuation in the network when $K=10$.

TABLE 1: Descriptive statistics of network topological structure.

\begin{tabular}{|c|c|c|c|c|c|}
\hline & & Mean & Min & $\operatorname{Max}$ & Std. \\
\hline \multirow{6}{*}{ Before market crash } & In-strength & 15.2488 & 0.0000 & 97.1489 & 11.8373 \\
\hline & Out-strength & 15.2488 & 0.0000 & 69.2445 & 10.7320 \\
\hline & Total strength & 30.4976 & 0.0000 & 118.6920 & 15.2375 \\
\hline & In-degree & 75.5393 & 0.0000 & 363.0000 & 47.8861 \\
\hline & Out-degree & 75.5393 & 0.0000 & 289.0000 & 45.5564 \\
\hline & Total degree & 151.0786 & 0.0000 & 511.0000 & 70.5668 \\
\hline \multirow{6}{*}{ After market crash } & In-strength & 46.1407 & 0.0747 & 205.2302 & 42.9866 \\
\hline & Out-strength & 46.1407 & 0.3904 & 255.6046 & 45.3281 \\
\hline & Total strength & 92.2814 & 1.0327 & 282.0779 & 54.0209 \\
\hline & In-degree & 199.8357 & 1.0000 & 617.0000 & 145.2864 \\
\hline & Out-degree & 199.8357 & 2.0000 & 666.0000 & 148.3278 \\
\hline & Total degree & 399.6714 & 6.0000 & 1027.0000 & 201.8751 \\
\hline
\end{tabular}

2015. In this stage, the Shanghai Composite Index witnessed a steep fall, down to 2,964 from its highest point of 5,172 within two months. Stage 2, which covers August 25, 2015, to January 2, 2016, was, in fact, a stage of sharp shock overall. The first rebounding was recorded to 3,600 from 2,964, and then falling down to 2,638. In light of these fluctuations, the overall time window was divided into three stages, which are the first-order stage, that is, the bull market stage from November
17,2014 , to June 12, 2015; the second-order stage, that is, the bear market stage from June 12, 2015, to August 25, 2015; and the third-order stage, that is, that of sharp shock from August 25, 2015, to January 2, 2016.

From Figure 5, fluctuation tends to be gentle and converges with the passage of time, which indicates that $C_{t}$, which measures the fluctuation across the entire network, finally converges to a fixed value. The amplitude of the 

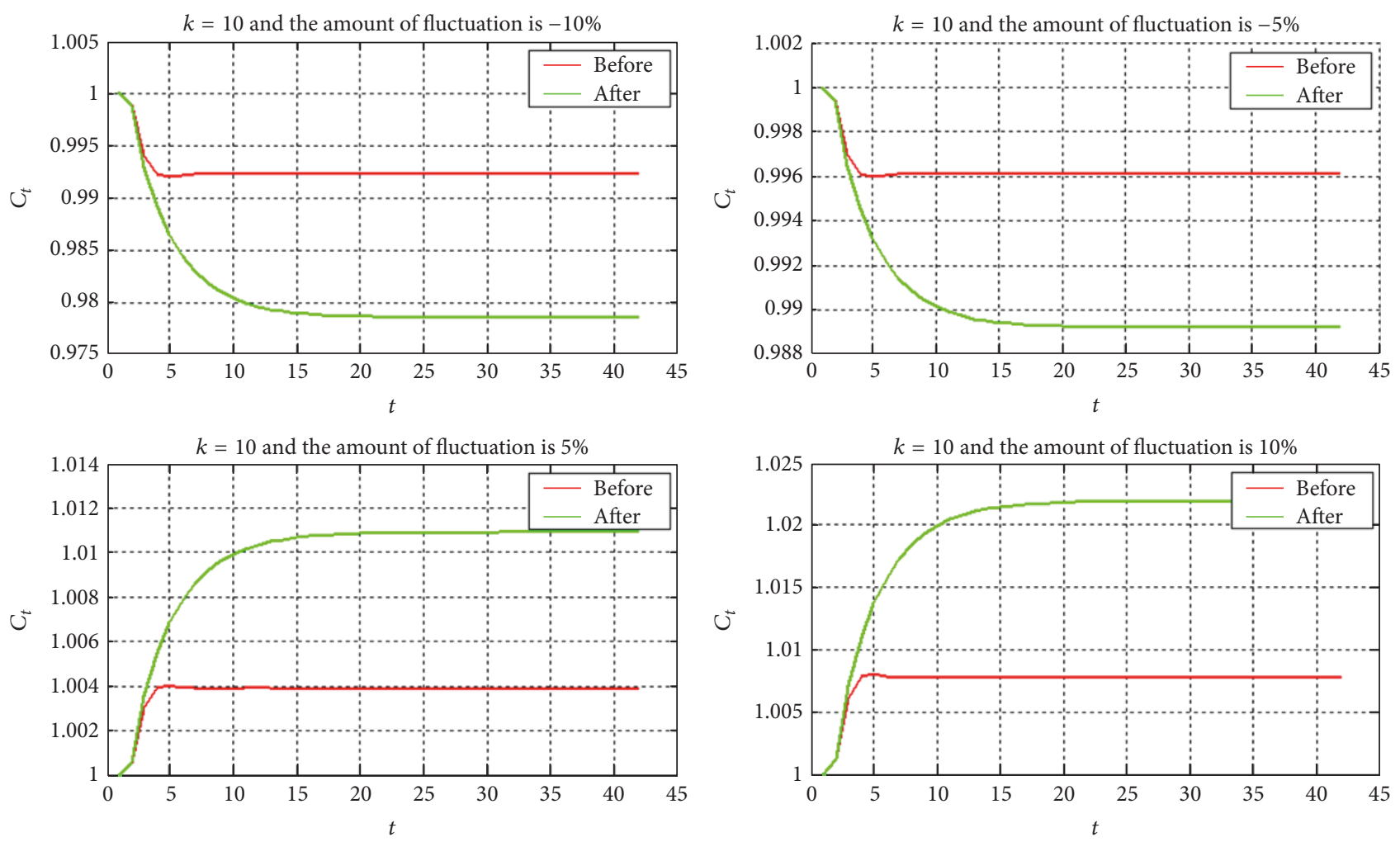

Figure 4: Influence of initial fluctuation on network stability when $K=10$.

fluctuation of stage 2, which corresponds to the period of steep fall, was the highest, followed by that of stage 1 before the stock market disaster, while the amplitude of fluctuation in stage 3 (the shock stage) was the lowest. When initial fluctuation was positive, the amplitude spread across the network was mostly positive, and vice versa. The latter results indicate that the direction of initial fluctuation determines the direction of the dynamic evolution of fluctuation across the entire network.

Based on Figure 6, the direction of initial fluctuation determines the direction of the continuous dynamic evolution of the network. A negative initial fluctuation can cause $C_{t}$ in the network to change to a negative direction, but a positive initial fluctuation can cause $C_{t}$ in the network to change to a positive direction. However, after a certain period, fluctuation tends to converge. In stages 1 and 3 , this convergence tended to occur after 6 to 7 iterations, and, in stage 2 , convergence tended to occur after 30 iterations. The results highlight that in the "shock" stage 3, the amplitude of change of $C_{t}$ in the network was the lowest. This result was followed by a rising amplitude in stage 1 , with the amplitude of change of $C_{t}$ in the network during the steep-fall stage 2 being the highest. This result indicates that, in the rising and shock stages, the network structure was dispersed and quickly digested external disturbance. Meanwhile, after the stock market disaster, in the steep-fall stage, the network structure seemed more tightly knit, but with an increased level of systematic risk as being unable to digest external disturbance quickly.

\section{Conclusions}

This paper selected a sample of 840 stocks from the Shanghai-Hong Kong Stock Connect market from November 17, 2014, to January 2, 2016. The study divided the time frame into two stages: stage 1 covered November 17, 2014, to June 12, 2015, as the bull market stage, and stage 2 from June 12,2015 , to January 2, 2016, as the bear market stage. Subsequently, stage 2 was further stratified into the steep-fall stage from June 12, 2015, to August 25, 2015, and the sharp shock stage from August 25, 2015, to January 2, 2016. This paper then employed cointegration because it is used in the traditional measurement method for building the directed weighted network of the stock market and for designing the indicators and value simulation for measuring network fluctuation. This step was conducted to study the dynamic evolution mechanism in a stock market transaction network during a period of fluctuation, thereby evaluating the topological structure and robustness of the network. Empirical results show the following. (1) In terms of the topological structure, the network structure in the bull market stage was found to be dispersed, and the network node degree distribution and strength distribution range were small, indicating that the generality and strength of the network's nodes were low and less influenced by or holding less influence over other nodes. Conversely, in the bear market stage, the network structure was closely knit, with a large node degree distribution and strength distribution range, indicating that the nodes in the network had a high degree and overall strength and were 

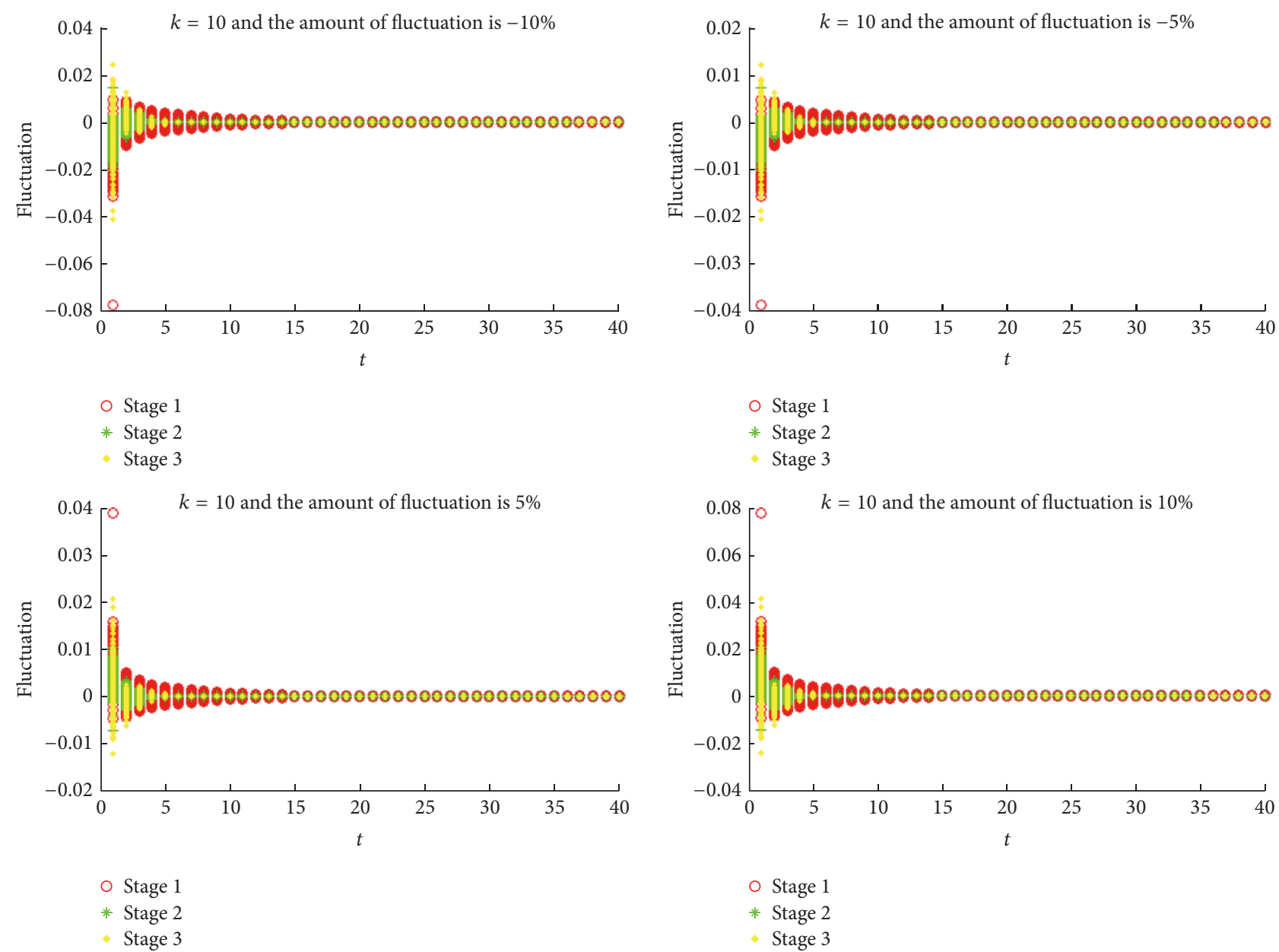

Figure 5: Dynamic evolution of fluctuation in the network when $K=10$.

both influenced by and could influence other nodes, to a great extent. (2) In terms of network stability, the network structure was strongly stable in the bull market stage. Under the initial given fluctuation, the dynamic evolution of the network converged rapidly and the amplitude was low. In contrast, in the bear market stage, the stability of the network structure was weak, with the dynamic evolution converging slowly under the fluctuation, and the amplitude of the fluctuation was high. (3) Then, by undertaking robustness analysis, the bear market stage was divided into the steep-fall and the sharp shock stages. Based on the analysis, in the steep-fall stage, the network structure was less stable, the fluctuation amplitude was very high, and a long period was needed prior to convergence.

The conclusions in this paper provide supplementary knowledge to the concept of stability in the traditional complex network theory. Traditional complex network analysis uses indicators to evaluate network connectivity. The higher the connectivity, the more stable the network and the higher its degree of robustness. Therefore, the closer the nodes are to each other in the network, the higher the degree of node and the bigger the weight of side will be. Moreover, the stability and robustness of the network will be higher. This research on stock market network structure demonstrates that network structure diversification corresponds to the diversification of systematic risk and thus helps in lowering systematic risk. A close network structure indicates that the diversification of systematic risk in the market is limited, and when systematic risk is high, convergence exists in the entire stock market. This result implies that when facing external disturbance, the fluctuation within the network will be high and risk communication will take a while to fade away.

\section{Disclosure}

Haifei Liu and Tingqiang Chen are co-first authors.

\section{Conflicts of Interest}

The authors declare that they have no conflicts of interest.

\section{Authors' Contributions}

Haifei Liu and Tingqiang Chen contributed equally to this work. 

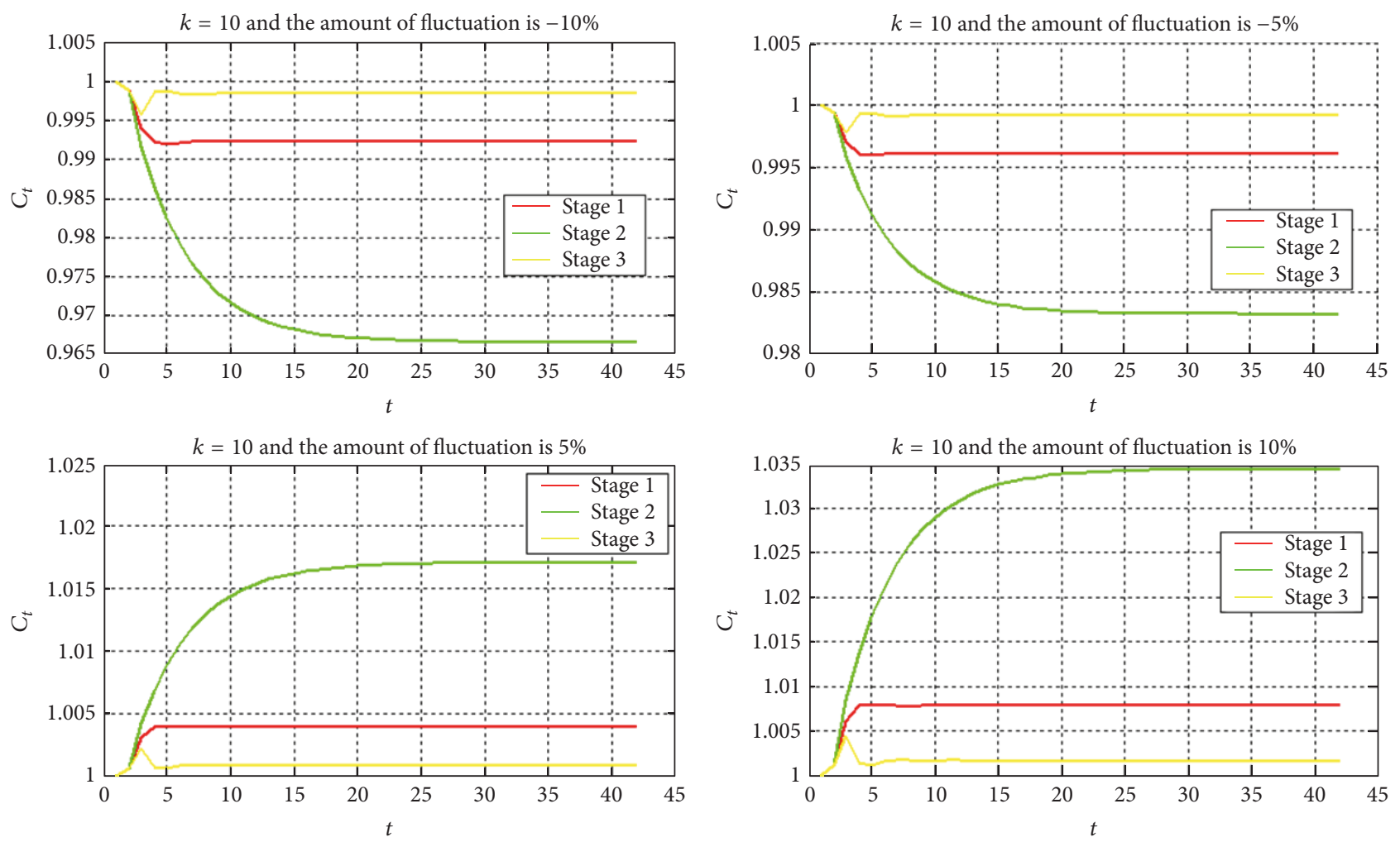

Figure 6: Influence of the initial fluctuation on network stability when $K=10$.

\section{Acknowledgments}

This work was supported by the National Natural Science Foundation of China (nos. 71771116, 71501094, 71501131, and 71720107001), the Natural Science Foundation of Jiangsu Province of China (nos. BK20150961, BK20161398), the Key Project of Philosophy and Social Science Research in Colleges and Universities in Jiangsu Province (no. 2017ZDIXM074), and Jiangsu Provincial Key Laboratory of Financial Engineering (no. NSK2015-09), the Fundamental Research Funds for the Central Universities (Project no. 011814380027), and the Qing Lan Project of Jiangsu.

\section{References}

[1] R. A. Hammond, Systemic Risk in the Financial System: Insights From Network Science, Pew Financial Reform Project, 2009.

[2] T. Chen, J. He, and X. Li, "An evolving network model of credit risk contagion in the financial market," Technological and Economic Development of Economy, vol. 23, no. 1, pp. 22-37, 2017.

[3] F. Kyriakopoulos, S. Thurner, C. Puhr, and S. W. Schmitz, "Network and eigenvalue analysis of financial transaction networks," Physics of Condensed Matter, vol. 71, no. 4, pp. 523-531, 2009.

[4] T.-Q. Chen and J.-M. He, "A network model of credit risk contagion," Discrete Dynamics in Nature and Society, vol. 2012, Article ID 513982, 13 pages, 2012.

[5] H. Liu, Y. Shen, W. Wang, and etc., "Network characteristics and influencing factors of beijing financial service sector cluster," Economic Geography, vol. 33, no. 1, pp. 131-137, 2013.
[6] X.-G. Yan, C. Xie, and G.-J. Wang, "The stability of financial market networks," EPL (Europhysics Letters), vol. 107, no. 4, Article ID 48002, 2014.

[7] T. Chen, X. Li, and J. Wang, "Spatial interaction model of credit risk contagion in the CRT market," Computational Economics, vol. 46, no. 4, pp. 519-537, 2015.

[8] L. Bakker, W. Hare, H. Khosravi, and B. Ramadanovic, "A social network model of investment behaviour in the stock market," Physica A: Statistical Mechanics and Its Applications, vol. 389, no. 6, pp. 1223-1229, 2010.

[9] S. Cai, L. Hong, and Z. Fu, "Complex Network Based Empirical Research on Financial Market's Network Structure," Complex System and Complexity Science, vol. 08, no. 3, pp. 29-33, 2011.

[10] H. Han, L. Wu, and N. Song, "Random matrix-based financial network model," Chinese Journal of Physics, vol. 63, no. 13, pp. 431-440, 2014.

[11] M. Eryiǧit and R. Eryiǧit, "Network structure of crosscorrelations among the world market indices," Physica A: Statistical Mechanics and its Applications, vol. 388, no. 17, pp. 3551-3562, 2009.

[12] X. Gao, H. An, H. Liu, and Y. Ding, "Analysis on the topological properties of the linkage complex network between crude oil future price and spot price," Acta Physica Sinica, vol. 60, no. 6, Article ID 068902, p. 495, 2011.

[13] H. Yang, P. Fang, H. Wan, Y. Liu, and H. Lei, "Evolution characteristics of complex fund network and fund strategy identification," Entropy, vol. 17, no. 12, pp. 8073-8088, 2015.

[14] S. Rafael, "Visualizing stock-mutual fund relationships through social network analysis," Global Journal of Finance and Banking Issues, vol. 3, no. 3, pp. 8-22, 2009. 
[15] W. Huang, X. Zhuang, and S. Yao, "Analysis of topological nature and clustering structure of stock-correlated network in China," Journal of Management Sciences, vol. 21, no. 3, pp. 94103, 2008.

[16] Z.-Q. Jiang and W.-X. Zhou, "Complex stock trading network among investors," Physica A: Statistical Mechanics and Its Applications, vol. 389, no. 21, pp. 4929-4941, 2010.

[17] T. Wang, "Research on statistical characteristics of weighted complex network of the second board in china's stock market," Journal of Xinzhou Teachers University, vol. 31, no. 5, pp. 1-5, 2015.

[18] T. Chen, Y. Chen, and X. Li, "An entropy model of credit risk contagion in the CRT market," in Discrete Dynamics in Nature and Society, p. 8, 2015, http://dx.doi.org/10.1155/2015/397852.

[19] S. Li and J. Li, "Social network structures and bank runs," The European Physical Journal B, vol. 89, no. 5, article no. 116, 2016.

[20] S. Li and X. Sui, "Contagion risk in endogenous financial networks," Chaos, Solitons \& Fractals, vol. 91, pp. 591-597, 2016.

[21] W. K. Winecoff, "Structural power and the global financial crisis: A network analytical approach," Business and Politics, vol. 17, no. 3, pp. 495-525, 2015.

[22] M. Tumminello, T. Di Matteo, T. Aste, and R. . Mantegna, "Correlation based networks of equity returns sampled at different time horizons," Physics of Condensed Matter, vol. 55, no. 2, pp. 209-217, 2007.

[23] W.-Q. Huang, X.-T. Zhuang, and S. Yao, "A network analysis of the Chinese stock market," Physica A: Statistical Mechanics and its Applications, vol. 388, no. 14, pp. 2956-2964, 2009.

[24] E. M. H. Lin, E. W. Sun, and M.-T. Yu, "Systemic risk, financial markets, and performance of financial institutions," Annals of Operations Research, vol. 88, no. 12, pp. 1-25, 2016.

[25] S. Vitali, S. Battiston, and M. Gallegati, "Financial fragility and distress propagation in a network of regions," Ssrn Electronic Journal, vol. 62, pp. 56-75, 2012.

[26] C. Yang, Y. Chen, L. Niu, and Q. Li, "Cointegration analysis and influence rank - A network approach to global stock markets," Physica A: Statistical Mechanics and Its Applications, vol. 400, no. 4, pp. 168-185, 2014.

[27] C. Tu, "Cointegration-based financial networks study in Chinese stock market," Physica A: Statistical Mechanics and Its Applications, vol. 402, no. 3, pp. 245-254, 2014. 


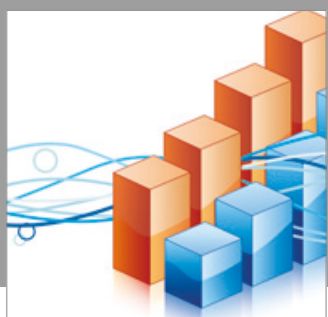

Advances in

Operations Research

vatersals

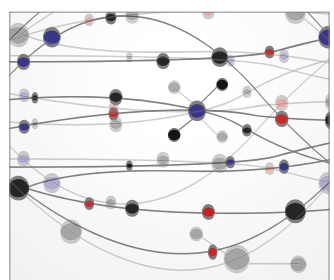

\section{The Scientific} World Journal
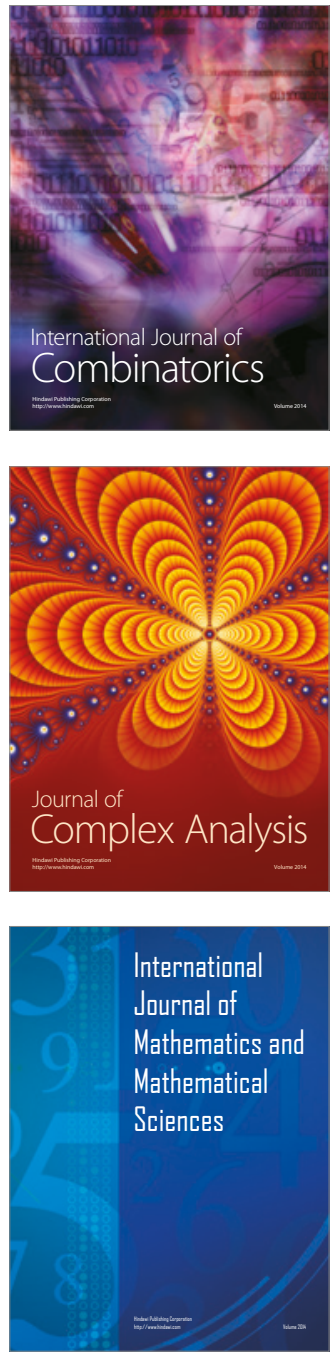
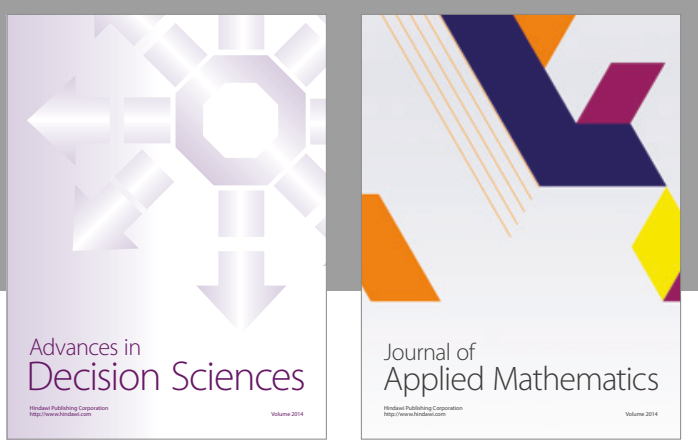

Algebra

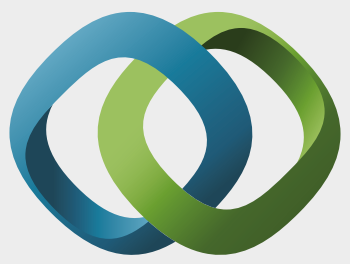

\section{Hindawi}

Submit your manuscripts at

https://www.hindawi.com
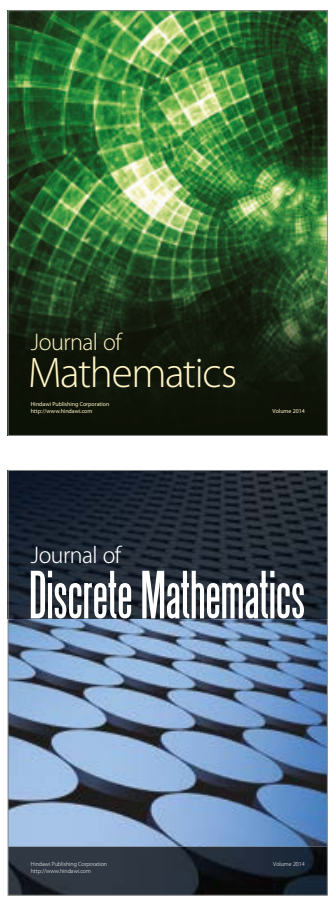

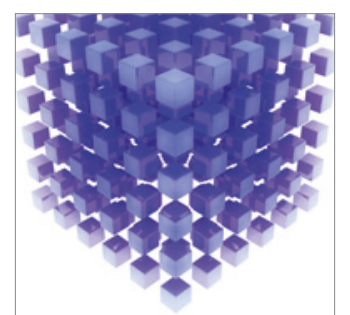

Mathematical Problems in Engineering
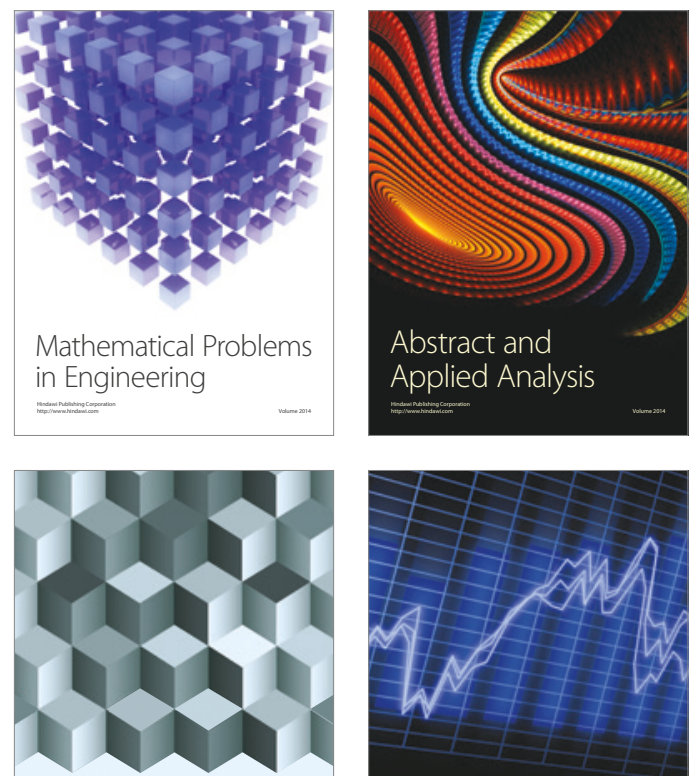

Journal of

Function Spaces

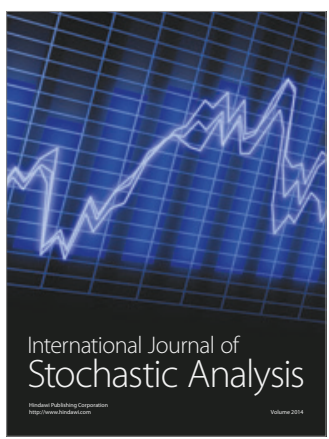

Probability and Statistics
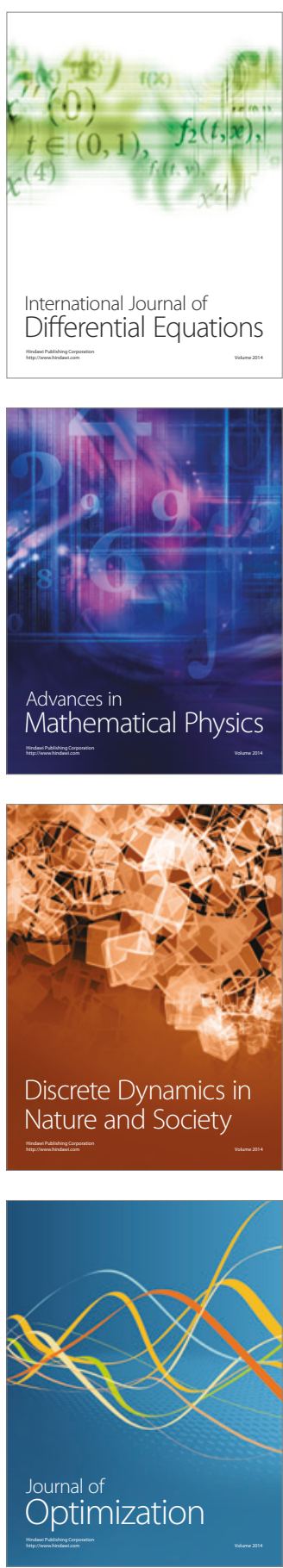